\title{
Selection of the optimal constellation of hybrid systems for pre-stressing
}

\author{
Slađana Miljanovic ${ }^{1, *}$ and Muhamed Zlatar ${ }^{2}$ \\ ${ }^{1}$ University of Sarajevo, Faculty of Architecture, Sarajevo, Bosnia and Herzegovina \\ ${ }^{2}$ University of Sarajevo, Faculty of Civil Engineering, Sarajevo, Bosnia and Herzegovina
}

\begin{abstract}
Hybrid systems, which were created by integrating the structural systems of different behavior in the transfer of loads, represent a new efficient system, with low self-weight, increased capacity characteristics and reduced deformability compared to the systems of which they are made of. Applicable to all materialization and logical geometric forms, they represent transparent systems suitable for optimization of structures. In this paper is represented the original analysis of parameters, reduced to relative values of a hybrid system with and without pre-stressing of different geometric shapes, particularly in terms of deformability. The results of the research are represented through the practical expressions and diagrams for use in practice, and on the basis of which can be determined suitable constellation of system from a simple girder, strut type hybrid system with different geometrical characteristics and pre-stressed hybrid system, and in the case of wood-steel hybrid system for different percentage of moisture of wood girder. To give access presents the accurate method that can be applied to any combination of materials in the context of a hybrid system.
\end{abstract}

\section{Introduction}

The optimal structural form created in "self-forming "process, representing a geometric review of the shortest loads transfer distance, and using an appropriate materials, they possess the optimal power of energy consumption in transfer of the load. The emergence of modern materials and the possibility of their combining set a condition to adapt the structural system by requirements of geometry, mechanics, features, loads, material properties and form. For the further development of structural system is imposed the complex task of achieving the mutual dependence of the geometric layout of the structure in the form, with the possibility of receiving mechanical loads depending on the type of the materials. Thereby structural elements are grouped according to the behaviour when carrying loads in a geometric series that provides sufficient capacity and functionality of structures and optimization of material consumption. The solution for set requirements is found in the development of the hybrid systems that offered the possibility of changing the form and behaviour, as well as increasing the capacity of the "pure" systems from which they are incurred[1,2].

*Corresponding author: sladjanam@af.unsa.ba 


\section{$2 \mathrm{Hybrid}$ systems}

Strut type hybrid system is formed by constructive uniting of two "clear" systems, or by girder which has flexural stiffness and by cable with stretch rigidity, with common points of reliance at the end of the girder. Their constructive unifying provide vertical struts which has infinite stiffness, are hinged to a solid girder.

\subsection{Parametric analysis from the aspect of capacity according to [1]}

The effects of parametric variations at the capacity of hybrid system, shown in general at carrier of a simple beam system in the action of constant load, and by bringing into connection the cross section forces with individual parameters.

\subsubsection{The impact of the number of struts - geometric forms of cable}
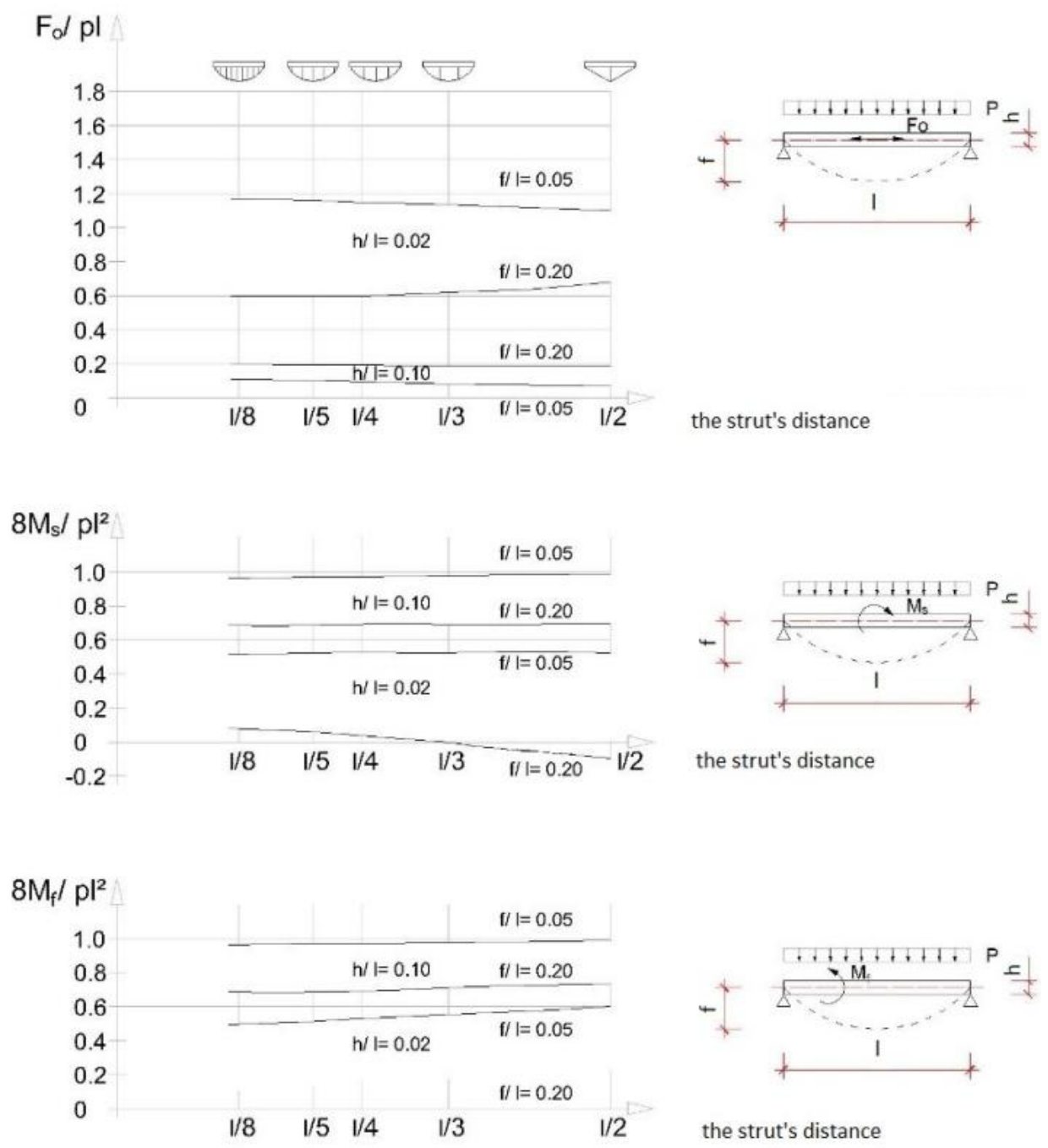

Fig. 1Dependence of the cross-section forces of form and stiffness of the hybrid system[1] 
The analysis examined changes in cross-section forces in relation to: the number of struts, ratio of arrow and span and the ratio of the height of the girder and span. The constant values are: span, distance of the struts, given as part of the span, the type of load, the ratio of cross-section surfaces of system elements and cross-sectional shape of girder. At Fig. 1 are given diagrams of ratio of forces in typical cross-section of girder for strut type hybrid systems of various geometric forms, depending on the number of struts, and in relation to the maximum impacts on simple beam carrier loaded by even load. Using the diagram can be concluded that for solid girders the number of struts does not affect the transfer of the load, while for the low height girder and larger arrows $(f / l=0,2)$ differences in extreme moments are evident, where for the single-strut type hybrid system moment becomes negative value for the cross-section above the strut.

\subsubsection{The impact of a combination of materials}

On the behaviour of hybrids when carrying loads, in addition to the geometric performance, the impact also has the differences of mechanical properties of materials from which their elements were derived. Behaviour of hybrid system is characterized by redistributing the load and by mode of deformation, which directly depends of the stiffness, or modulus of elasticity of the embedded materials.

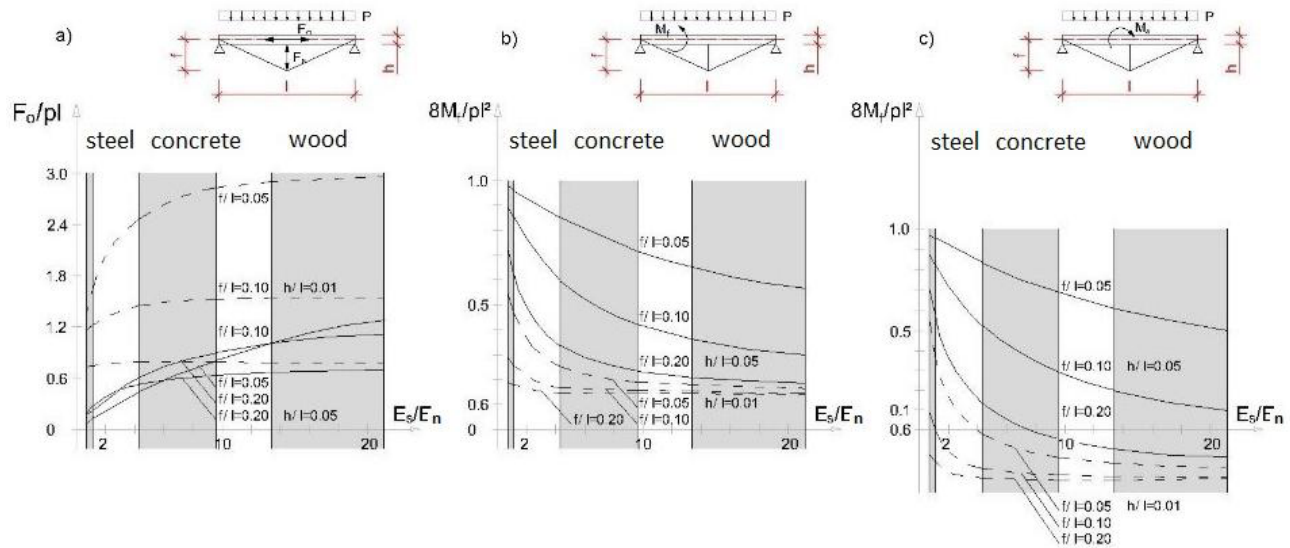

Fig. 2The impact of combination of materials on cross-sectional forces of hybrid system expressed by relation of modules of cable's and girder's elasticity: a)normal forces in girder b) moment in field c) moment above the strut [1]

At Fig. 2 are shown changes in redistribution of load for different relations of modulus of cable's and girder's elasticity. For small geometric height of hybrid, the force of pressure in girder rapidly grows in stiffer girder, while in small stiffness receives a constant stream. Then bending moments above the strut for rigid girders for all geometric heights and solid girders have positive sign. For girders of smaller stiffness of material this value drops sharply, and for large geometric height and small stiffness of girder achieves a negative sign. 


\section{Pre-stressed hybrid systems}

\subsection{Impacts on the hybrid system's constellation for pre-stressing from the standpoint of capacity}

The characteristics of the hybrid system suitable for pre-stressing from the standpoint of capacity according to[1], are:

- affects the number of struts, with or without pre-stressing;

- exclusively depends on the ratio of girder's stiffness for bending and stretching cables;

- changing the girder's cross-section from rectangular through the segmented (I profile), rapidly decreases the rigidity of the cable, while the rigidity of the girder seamlessly drops.

Pre-stressing of hybrid systems is one of the ways to increase the rigidity of the whole system. Pre-stressing is effective only when the hybrid system composed of the bar elements, wherein the resistivity of a cable system to the normal force is in balance with the resistance to bending of the girder. According to [1], in hybrid systems, where is more equal redistribution of loads between the girder and cable, later addition of the pre-stressing force gives positive effects.

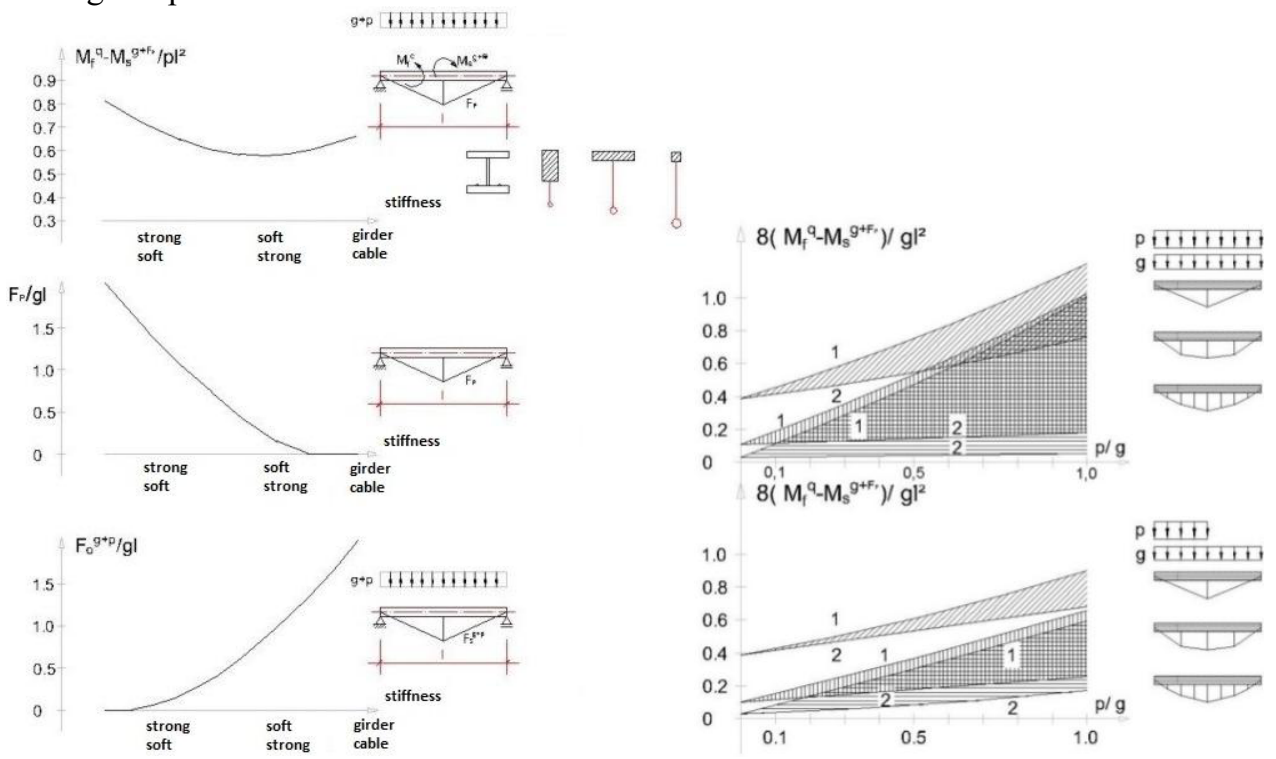

Figure 3 a) Utilization of pre-stressed girder on the total load compared to a simply supported beam under constant load; b) Utilization of the bending moments, depending on the number of struts[1]

If we look at the hybrid systems with different girders stiffness, it can be concluded that:

- The uneven distribution of moments is expressed at very solid steel girders, as well as for soft wooden girders inside the hybrid system with a marked rigidity of supporting cable;

- Pre-stressing efficiently causes an increase in the rigidity of the system and for uniform distribution of bending moments under various schemes of the load, which is better the mutual compatibility of partial rigidity. The more number of struts the more balanced utilization of bending moments is (Fig. 3b). 


\subsection{Own parametric analysis from the aspect of deformability}

\subsubsection{The rigidity of the strut-type hybrid systems for external load $q$}

The rigidity of the hybrid system consists of partial stiffness, namely: rigidity to the elastic deformation of the individual elements of the hybrid and rigidity dictated by the geometric arrangement of the system's elements in relation to the reception of load $[3,4]$.

Table 1.Geometric stiffness of hybrid systems with different geometrical forms[3]

\begin{tabular}{|c|c|c|}
\hline $\begin{array}{l}\text { Geometrical } \\
\text { form }\end{array}$ & $\Delta f$ & $K_{G, S}$ \\
\hline 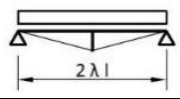 & $\frac{F_{S, p}^{H I I}}{E_{S} \cdot A_{S}} \cdot f \cdot G=\frac{q \cdot l^{2}}{8 f \cdot E_{S} \cdot A_{S}} \cdot f \cdot \frac{l^{2}}{4 f^{2} \cdot \cos ^{3} \alpha}$ & $32 f^{2} \cdot \cos ^{3} \alpha \cdot E_{S} \cdot A_{S}$ \\
\hline 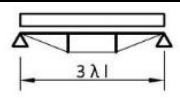 & $\frac{F_{S, p}^{H I I}}{E_{S} \cdot A_{S}} \cdot f \cdot G=\frac{q \cdot l^{2}}{8 f \cdot E_{S} \cdot A_{S}} \cdot f \cdot \frac{l^{2} \cdot\left(1+\cos ^{3} \alpha\right)}{9 f^{2} \cdot \cos ^{3} \alpha}$ & $\frac{72 E_{S} \cdot A_{S} \cdot f^{2} \cdot \cos ^{3} \alpha}{\left(1+\cos ^{3} \alpha\right)}$ \\
\hline 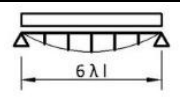 & $\frac{F_{S, p}^{H I I}}{E_{S} \cdot A_{S}} \cdot f \cdot G=\frac{q \cdot l^{2}}{8 f \cdot E_{S} \cdot A_{S}} \cdot f \cdot\left(1+\frac{3}{16} \cdot \frac{l^{2}}{f^{2}}\right)$ & $\frac{128}{3} \cdot f^{2} \cdot E_{S} \cdot A_{S}$ \\
\hline
\end{tabular}

For the purposes of testing the load redistribution are derived expressions in Table 1. For $K_{G, S}$ whose rigidity of cable within hybrid systems of different geometrical forms (such as single, double and multiple strut, respectively) and which can be used to study the behaviour of the hybrid system under load. The geometric characteristic $G$ was used in addition to the possibility of describing the geometric parameters of stiffness even for budget of cross-sectional size and strain on the theory of a higher order[3, 4].

\subsubsection{Redistribution of load within hybrid system}

Reducing the deformation of the hybrid system in relation to the beam with rigidity for bending is caused by the activation of the cable consisting of geometric stiffness and tensile stiffness. Deformability of the hybrid system can be analysed by observing the hybrid system in borderline cases of deformability of individual systems that make up. The labelled sections were observed in those that provide the connection between the individual basic systems, or sections where it can be established the compatibility of deformations of the individual systems in the hybrid system[5].If the hybrid system is composed of elements of limited stiffness in bending and stretching, then the order of transfer of the load, or strain of the system as follows:

$$
\omega_{h y b}\left(q_{u k}\right)=\omega_{n}\left(q_{u k}\right)-\omega_{n}(V)+\Delta f
$$

where $\omega_{\text {hyb }}\left(q_{u k}\right)$ - deflection of hybrid system in a characteristic cross-section of the strut connection, $\omega_{n}\left(q_{u k}\right)$ - deflection of girder of the total load with flexible cable, $\omega_{n}(V)$ deflection of girder in a characteristic cross-section of the total activated forces in strut of the unyielding cable and $\Delta f$ - vertical deformation of the cable due to full force of pressure in strut.

If this approach is applied for different geometric forms of hybrid systems with elements of limited rigidity of system, it is possible to obtain exact expressions for finding forces in strut and deflection values of the hybrid system. Then, the single strut-type hybrid system loaded with the external load q, the value of the real force in the strut, and real 
deflection in the characteristic section according to the Table 2. The condition $V_{h y b, s t v}=0$ practically means that the strut has no influence on the deformability of the system. In this case the requirement meets the tie partial of stiffness, bending beams and cable strength in its geometric form, which means that for equal redistribution of loads between girders and cable, the hybrid system should pre-stress to reduce the deformability[3].

Table 2The value of the real force in the strut, and real deflection in the characteristic section

\begin{tabular}{|c|c|c|}
\hline $\begin{array}{l}\text { Geometrical } \\
\text { form }\end{array}$ & $V_{h y b, s t v}\left(q_{u k}\right)$ & $\omega_{h y b}\left(q_{u k}\right)$ \\
\hline 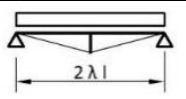 & $\frac{q_{u k} \cdot l^{2} \cdot \sin \alpha}{4 f}\left(1-\frac{48 E_{n} \cdot I_{n}}{32 f^{2} \cdot \cos ^{3} \alpha \cdot E_{S} \cdot A_{S}}\right)$ & $\frac{5}{384} \cdot \frac{q_{u k} \cdot l^{4}}{E_{n} \cdot I_{n}}-\frac{V_{h y b, s t v} \cdot l^{3}}{48 E_{n} \cdot I_{n}}$ \\
\hline 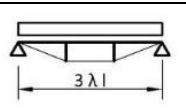 & $\frac{q_{u k} \cdot l^{2} \cdot \sin \alpha}{8 f} \cdot\left(1-\frac{9\left(1+\cos ^{3} \alpha\right) \cdot E_{n} \cdot I_{n}}{40 f^{2} \cdot \cos ^{3} \alpha \cdot E_{S} \cdot A_{S}}\right)$ & $\frac{11}{972} \cdot \frac{q_{u k} \cdot l^{4}}{E_{n} \cdot I_{n}}-\frac{5 V_{h y b s t v} \cdot l^{3}}{162 E_{n} \cdot I_{n}}$ \\
\hline 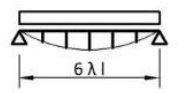 & $\frac{q_{u k} \cdot l^{2}}{4 f} \cdot \sin \alpha_{3} \cdot\left(1-\frac{270 E_{n} \cdot I_{n}}{176 f^{2} \cdot E_{S} \cdot A_{S}}\right)$ & $\frac{5}{384} \cdot \frac{q_{u k} \cdot l^{4}}{E_{n} \cdot I_{n}}-\frac{176}{2304} \cdot \frac{V_{h y b, s v} \cdot l^{3}}{E_{n} \cdot I_{n}}$ \\
\hline
\end{tabular}

Based on this approach to work will continue to be implemented parametric analysis of a hybrid girder with the effects of pre-stressing.

\subsection{Selection of the optimal constellation of hybrid system in terms of deformability in the case of glulam-steel material combination}

For analysis of the initially taken constant parameters $E_{n} / E_{S}$, and for the different forms of the hybridgirder are varied $f / l, h / l$ and $A_{n} / A_{S}$. The ordinate of the diagram shown at the Fig. 4 , is given the relative value of the force in the strut to the total load of the system, and the various forms of the system, which is shown on the abscissa and the calculated values of the mutualspacingaid system.
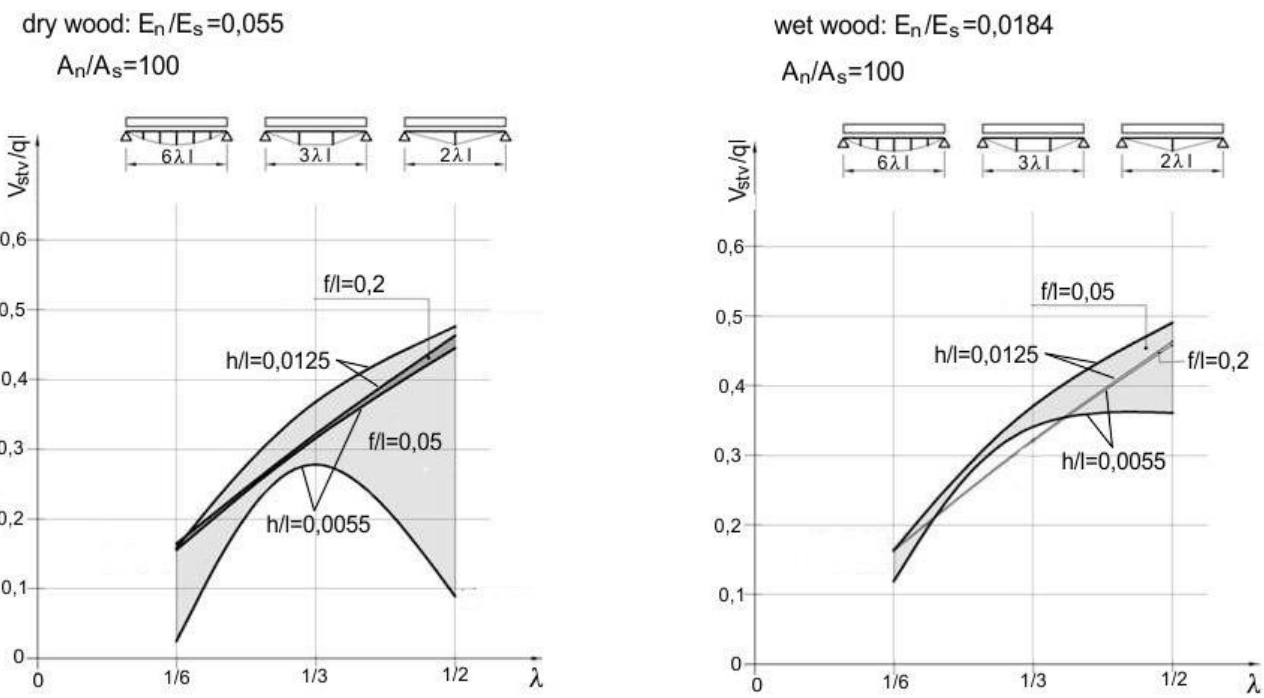

Figure 4 Size of relative pressure force in strut of the maximum deflectiondepending on the number of struts: a) dry wood, b) wetwood 
Based on diagramsit's for small geometric height of the hybrid system of these forms the greatest effect of cable-supporting is achieved by a single-strut hybrid system, and given the number of struts and the way of redistribution of loads. Also, single-strut hybrid system has the highest sensitivity to changing geometric height of hybrids and stiffness in bending girders.

dry wood $E_{n} / E_{s}=0,055$

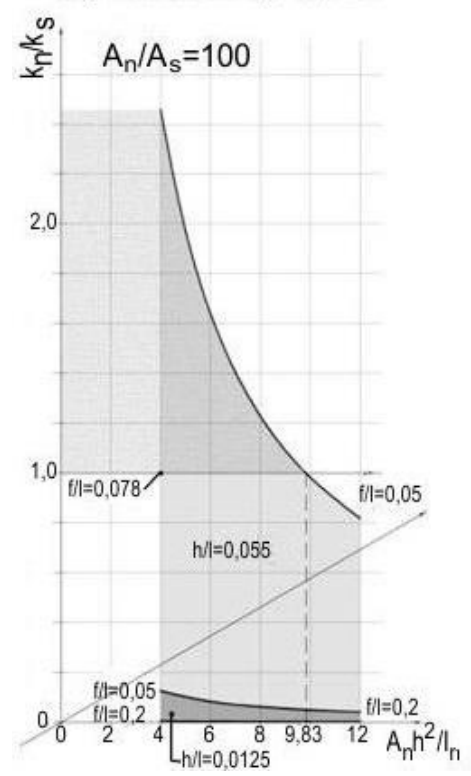

wet wood $E_{n} / E_{s}=0,0184$

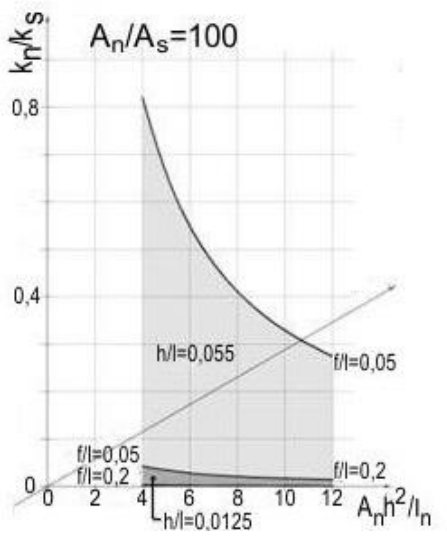

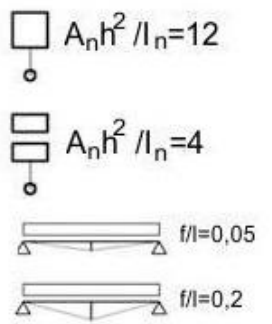

Figure 5 The relationship of the individual stiffness for effect of activated forces in strut

Further analysis was conducted for single-strut hybrid system evenly loaded, to change cross-sectional shape of wooden beams (Figure 5). Subscriptions are sized for relative force of pressure in strut, and for limited values of the height of the girder and the geometric height of the hybrid system, for particular relations between cross sectional area of the girder and the cable (shown in the applicator), and in particular discussed changes in the modulus of elasticity of the wooden beams due to increase in the percentage of moisture content. In parallel with this analysis is provided an overview of the relationship of the individual stiffness, for the effect of activated forces in strut, girder of the bending and stretching of the cable in the characteristic shape of the hybrid system, which is given on the vertical axis of the diagram, while the $\mathrm{x}$-axis shows the ratio of positional and its own moment of inertia, and for square and separated cross-section.For single-strut hybrid system the expression for the ratio of the beam's stiffness and cable to the effect upon activated compressive force in strut are given in Eq.2.

$$
k_{n}(V) / k_{S}(V)=\frac{48 E_{n} \cdot I_{n}}{32 f^{2} \cdot \cos ^{3} \alpha \cdot E_{S} \cdot A_{S}}=\frac{3}{2} \cdot \frac{E_{n}}{E_{S}} \cdot \frac{I_{n}}{A_{n} \cdot h^{2}} \cdot \frac{A_{n}}{A_{S}} \cdot \frac{h^{2}}{l^{2}} \cdot \frac{l^{2}}{f^{2}} \cdot \frac{1}{\sqrt{\left(\frac{4 f^{2}}{l^{2}}+1\right)^{3}}} \leq 1
$$




\section{4 The effective force of pre-stressing}

For finding the value of effective force of pre-stressing is used the assumption that with the pre-stressing of wooden beams with lower quality class may defer the failure of crosssection in bending, or reducing the variability of flexural strength $[3,6,7,8,9,10]$.

For finding the size of the effective pre-stressing force to reduce the deformability of the hybrid system it is necessary to re-observe the system in borderline cases of deformability of the individual elements that make up, due to the fact of only the pre-stressing force to the system. The characteristic observed sections are those that provide the connection between the individual basic elements, or sections in which can be made compatibility of single "clean" system's deformation within the hybrid system[3].
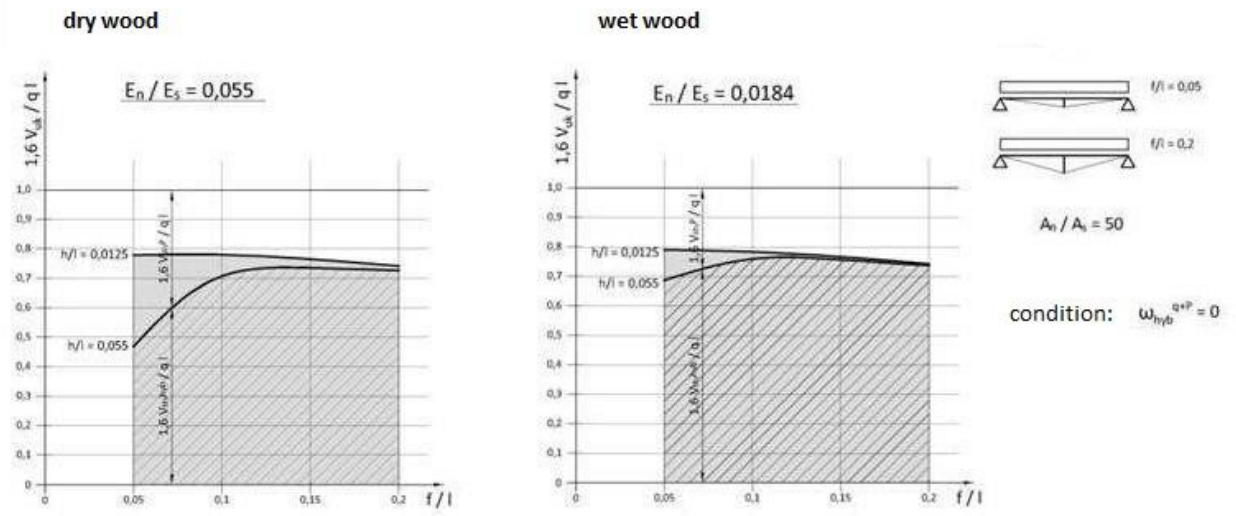

Fig. 6 The real values of pressure forces in strut by external load and necessary value of force in strut by pre-stressing

If the hybrid system is burdened only with the pre-stressing force entered in the cable by stretching, activates the pressure force in strut which causes vertical deformation of beam and cable. If these deformations are observed in cases of extreme behaviour of the two pure systems depending on the stiffness, obtained a direct dependence of the size of the cable's elongation and deflection of the hybrid system. The dependence of the cable's elongation and deflection of the single-strut type of hybrid system are given in Eq.3:

$$
\delta=2 \sin \alpha \cdot \omega_{n} \cdot\left(1+\frac{48 E_{n} \cdot I_{n}}{16 f^{2} \cdot \cos ^{3} \alpha \cdot E_{S} \cdot A_{S}}\right)
$$

At Fig. 6 are provided real values of force in strut of pre-stressed hybrid system loaded with an even load q, such as the values of the real forces in strut for external load (Table 2.), and the value of force in strut of that should be achieved with pre-stressing makes the difference to the full values.

\section{Conclusions}

For all of the analysis changes in the behaviour of different forms of hybrid system, with the same characteristics of the cross-section beams, geometric height and appropriate relations area of cross-sectional elements of the hybrid can be concluded as follows: 
- From the aspect of carrying the load, constellation of hybrid system suitable for prestressing are those in which happens equalisation of moment at girder with rapid increase of normal pressure force(Fig.2);

- Pre-stressing is suitable for hybrids with wooden girder, where is achieved reduction of variability of wood bending strength, or concrete girder, where the effect of pressure force is dominant;

-For hybrids of small geometric height with increasing the rigidity of the girder, increasing the height of the cross-section or parsing cross-section beams, the cable does not have a sufficient role in carrying the load. Then, for all forms of hybrid systems can be applied pre-stressing to increase the stiffness of the cable in the system and more correct redistribution of the load on the system's elements, given in the example of hybrid systems with a girder of glulam timber and steel cable with effective force of pre-stressing;

-For tested forms of hybrid systems in which is small geometric height the highest sensitivity when changing relations of stiffness and highest deformability shows the singlestrut system.

\section{References}

1. R.Wagner, K.Gabriel, J.Schlaich, Hybride Tragwerke (Die logische Erfassung entwurfsrelevanter Faktoren: Geometrie-Funktion-Last-Auflager-Werkstoff-Form).Stuttgart: Institut fur Massivbau, Universitat Stuttgart. (Schl 172/13-1).

2. S. Miljanović, External Pre-stressing of the Glued Laminated Timber Beam in Real Conditionsdoctoral dissertation. Sarajevo: Faculty of Architecture, (2012).

3. S. Miljanović, M. Zlatar, The conceptual design of hybrid structures - theoretical and experimental research of external prestressed timber beams, Structures and Architecture, ch. 182, pg. 1499-1506 (Taylor \& Francis Group, 2016).

4. S. Miljanović, M. Zlatar, Theoretical and experimental research of external prestressed timber beams in variable moisture conditions, CSM,4, No. 2,191-209, (2015)

5. S.Miljanović, M. Zlatar, Parametric analysis of prestressed hybrid systems, Proceedings of the 8th International Congress of Croatian Society of Mechanics, (Centr al European Association for Computational Mechanic,Opatija, 2015).

6. J.H.Ahn, C.Y.Jung,S.H. Kim, Structural Engineering and Mechanics, 34(2), 247-275, (2010).

7. B. G. Ansharia, Construction and Building Materials, 29, 24-32, (2012).

8. B. F. Deam,Journal of Structural Engineering, 134(5), 801-809, (2008).

9. A. H. Ibrahimbegović, Linear instability or buckling problems for mechanical and coupled thermomechanical extreme conditions'. CSM, 2, 349-374, (2013).

10. M. Yahyaei-Moayyed,Construction and Building Materials, 25, 2495-2506, (2011). 